\title{
Ophthalmology and general medicine
}

\author{
Alec Karseras
}

This ophthalmological perspective of general medicine attempts to deal with the more common situations encountered clinically in general ophthalmic practice. Some priority has been given to the clinical usefulness of data. A comprehensive review would involve a wide range of rare conditions and has not been attempted. However, some mention of rarities is made in the context of important differential diagnoses.

The emphasis on vascular phenomena and somatisation syndromes reflects their preponderance in clinical practice.

Pathology of the peripituitary area, although important, is comparatively uncommon in ophthalmological practice. This and other affectations of the central nervous system giving rise to the subspecialty of neuroophthalmology, are not included in this review.

\section{Headache}

Headache is a common, if not the commonest presentation symptom in clinical practice. A considerable number of patients with this symptom are referred to ophthalmologists. Ostensibly this is to exclude an ophthalmic cause. Without a relationship to prolonged use of the eyes for close work or a struggle to overcome diplopia, it is unlikely that an ocular causation will be present. Facultative hypermetropia, convergence insufficiency, esophorias and most exophorias (the tendency for the eyes to converge or diverge respectively) all have a relationship to use of the eyes especially for close work.

Sometimes, patients with headaches are referred for an opinion on the optic discs in order to exclude the possibility of papilloedema from raised intracranial pressure. High hypermetropia, buried drusen (see below) and familial pseudopapilloedema may all mimic the oedema of raised intracranial pressure (plerocephalic oedema). If venous pulsation is present at the disc head the clinician can be reasonably assured that there is no raised intracranial pressure causing plerocephalic oedema of the disc.

Buried drusen usually have a tell tale sparkling glint at the disc head and are small nodules of tissue with a specific histology.

Department of

Woolos Hospital,

Gwent Healthcare

NHS Trust, Newport,

Gwent,UK

Correspondence to:

Mr A Karseras, The Laurels,

27 Penlline Road,

Whitchurch, Cardiff

CF14 2AA, UK

Submitted 8 February 1999 Accepted 27 October 1999 five to 20 minutes, separates migrainous photopsiae from the lightning-like flashes of vitreoretinal traction which are of possible preretinal detachment significance.

Transient hemianopic or altitudinal visual field loss, whether uniocular (retinal) or binocular (cerebral) is more likely to have a migrainous vasospastic cause in young healthy adults than organic vaso-occlusive phenomena (see below). However, peripituitary space occupying lesions occasionally give a similar intermittency and duration of visual or visual field loss.

The classical throbbing unilateral headache is not always apparent. The ache may bifrontal, uniocular, bitemporal, or occipital. Some patients' symptoms overlap with tension headaches or atypical facial pain. Various syndromes have been described centring around the distribution of pain. It is possible that most are the individuals' biological response (in the broadest terms) to their life situations. Their importance lies in recognising their nonorganic status, on clinical and historical criteria and in being selective regarding investigation.

\section{Organic based binocular and uniocular visual loss}

The nearer middle age progresses towards old age the greater the likelihood of an organic causation of occlusive vascular phenomena. Transitory uniocular visual loss (amaurosis fugax) lasting five to 20 minutes is mainly embolic from the carotid arteries with diabetes especially as a predisposing factor of presenile vascular disease.

Cardiac, paradoxical, fat or air emboli, etc, are extremely rare in comparison and may not be seen in a professional lifetime.

Rarely, migrainous spasm can result in infarction of the retina or visual pathways causing permanent uniocular or binocular homonymous visual field loss respectively.

When clinically obvious hypertension or diabetes is not the causative factor in permanent occlusive vascular disease in younger adults, inflammatory vascular disease (Behçet's, sarcoid, collagenosis) together with blood viscosity syndromes and thrombophilic conditions should be eliminated.

Symptomatic and asymptomatic emboli found in the retinal vessels prompt investigation for hypertension, diabetes, and hyperlipidaemia. Whether the emboli are fragmented platelet, refractile cholesterol, or junctional impacted thrombin, the source and cause must be pursued. Duplex scan of the carotids, blood and cardiac investigations may be indicated. The age and general status of the patient may determine the appropriate treatment, for example aspirin, lipid lowering measures, anticoagulants, or endarterectomy. 


\section{Temporal arteritis}

In the over 55 age group temporal arteritis may present with headaches and/or occlusive vascular disease.

Clinically the flat, pale blurring of the optic disc is pathognomonic of temporal arteritis. The multiple short posterior artery occlusions to the optic disc head do not allow any primary or secondary capillary congestion as in other disc oedemas.

The consideration of temporal arteritis in the differential diagnosis of amaurosis, temporal headaches, or acute visual loss in the elderly must be a priority. Prepresentation constitutional malaise, tenderness of temporal arteries, raised erythrocyte sedimentation rate and rarely, biopsy will usually exclude this potentially blinding and lethal condition.

\section{Basilar artery migraine}

Without the classical occipital headache this is a diagnosis which is frequently overlooked. It can present in younger patients with transitory diplopia, with or without vertigo, and/or bilateral amourosis. Myasthaenia gravis, demyelinating disease, and breakdown of phorias (the tendency to squint) can similarly give transient diplopias in young adults. Rarely, raised intracranial pressure without headache can give sixth cranial nerve paresis with transient diplopia but plerocephalic oedema will usually be evident.

The unwary may miss a uniocular diplopia resulting from a meibomian cyst of the upper lid.

\section{Painful third cranial nerve palsies}

An episode occurring some years previously will signify migraine as the cause. In the absence of a previous episode the patient must be deemed to have a leaking aneurysm until proved otherwise.

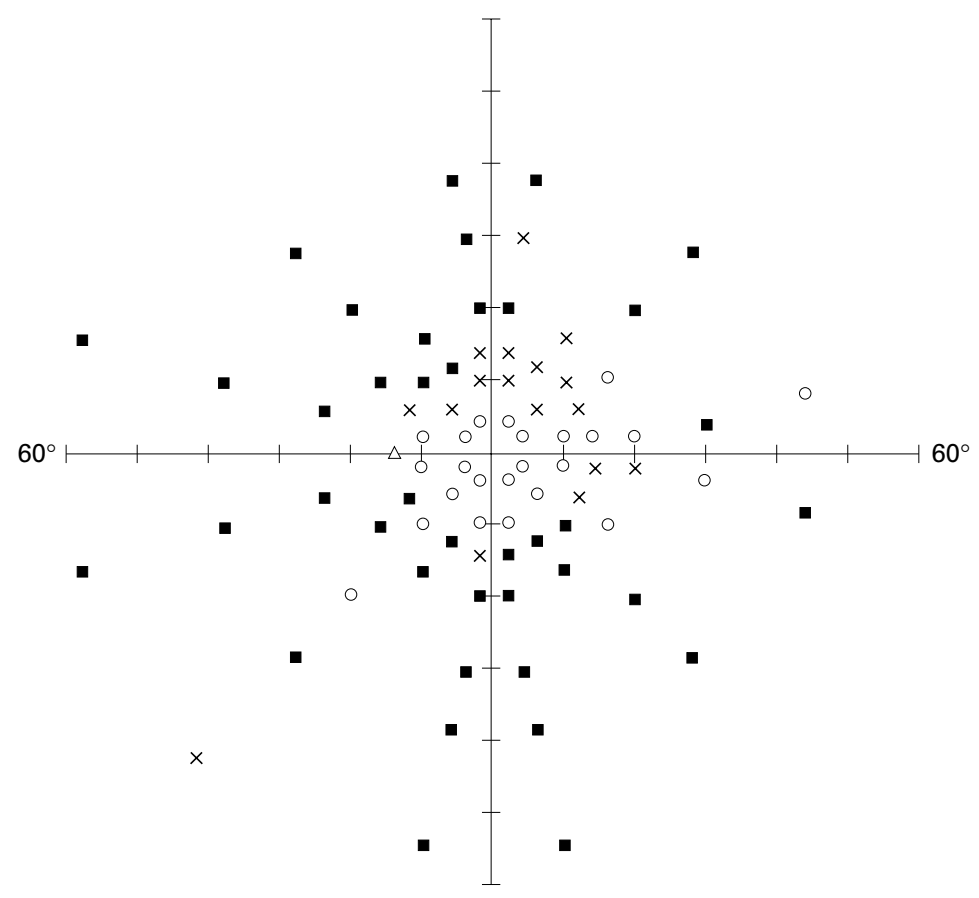

Figure 1 Computerised analysis showing widespread peripheral field loss.
Both conditions can give a recent onset of episodicity in the presence of a third cranial nerve palsy. If the pupil is spared it is unlikely that a space occupying lesion is afflicting the third cranial nerve. The lid is usually ptotic and the eye divergent.

Other causes of a painful third cranial nerve palsy are temporal arteritis, diabetes, and herpes zoster.

\section{Preocclusive vascular disease}

The role of ophthalmology in the diagnosis and management of target organ damage is now well established in diabetes. Criteria demanding tighter control of blood glucose, blood lipids, and blood pressure are often clinically evident on ophthalmoscopy.

Recently, the results of the hypertension optimal treatment randomised control trial have confirmed the experience of clinical ophthalmologists ${ }^{1}$ : namely that ophthalmoscopically evident peripheral vascular disease (grade I or II hypertensive arteriopathy) occurs at blood pressure levels deemed acceptable to general practitioners and physicians. Apparently, the British Hypertensive Society may revise the threshold for the initiation of treatment downward to $140 / 90$ when target organ damage is evident.

It will be an interesting prospect as to whether the appearance of retinal arterioles relative to the age of the patient, or retinal branch vein occlusions, become accepted criteria of end organ damage.

With target blood pressures envisaged at 140/80 such low pressures may be more easily attained if treatment is started early before extensive peripheral arteriolar structural changes have taken place.

Clinical appraisal of the retinal arterioles as an early indicator for treatment of hypertension may yet become more generally accepted.

The following case study illustrates a not uncommon situation. The patient was a male college lecturer, age 62 , who first presented in March 1996 with loss of vision in lower half of his left visual field. On examination it was found that he had left papilloedema with congestion and haemorrhage at disc head, inferior altitudinal field loss to confrontation, widespread peripheral field loss on computerised analysis (see fig 1), and bilateral retinal artery attenuation with irregular lumina. His blood pressure on ophthalmic presentation was 150/ $100 \mathrm{~mm} \mathrm{Hg}$ and erythrocyte sedimentation rate $8 \mathrm{~mm} /$ hour. Non-inflammatory anterior ischaemic optic neuropathy was diagnosed.

The patient was referred for consultant physician investigation. All investigations were found to be normal and no treatment was prescribed. The patient's blood pressure was recorded as $150 / 90 \mathrm{~mm} \mathrm{Hg}$. Two and a half years later the patient presented with identical clinical features in his second eye and he had lost his driving licence (see binocular fields in fig 2).

It would appear that some individuals are vulnerable to occlusive vascular disease with blood pressures regarded as "labile" or "white coat". Pressures previously deemed to be normal especially when there is absence of target organ 


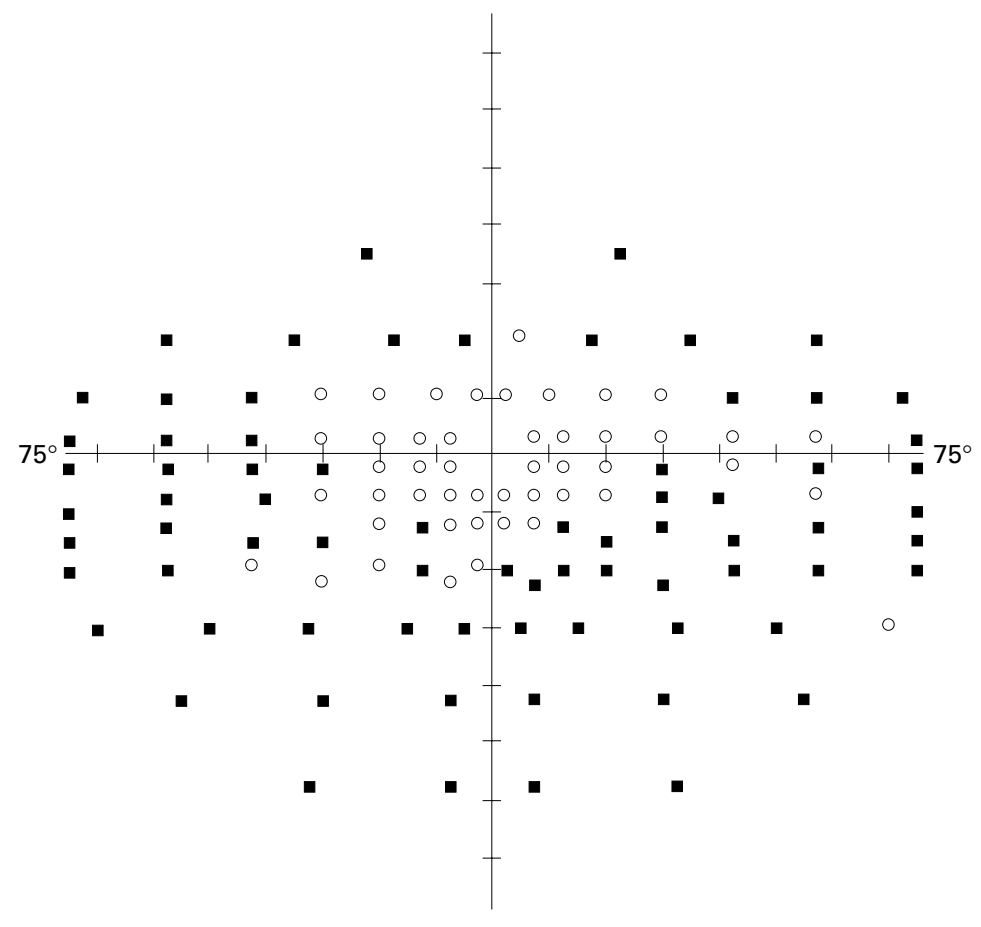

Figure 2 Binocular field loss. accompanied by incomplete closure of the eyes on blinking or when gently closed (lagophthalmos). This can cause irritable eyes and photophobia from punctate epithelial erosions of the cornea.

Lid retraction may be intermittent or demonstrable during vertical eye movements (lid lag). Sclera showing below the cornea indicates proptosis with the potential complications of diplopia, optic nerve threat, and exposure keratopathy. All such exophthalmos should be reviewed by ophthalmologists. Therapy may involve protective drops, medical or surgical upper lid lowering procedures, tarsorrhaphy, medical or surgical decompression.

Bilateral lid retraction may be seen also in some anxiety states and mid-brain disorders, for example, Parkinson's disease.

Where diplopia is a prominent symptom, especially if the pattern of extraocular paresis does not fit with dysthyroidism and/or there is little or no proptosis, associated myaesthenia gravis should considered.

\section{Somatisation syndromes}

As in all other areas of medicine, ophthalmology experiences many patients with symptoms for which a peripheral organic cause is absent. Pain or varying levels of discomfort are the commonest presentations. Recent onset of symptoms in a stable personality should "ring bells". The uniocular pain of acute sinusitis, retrobulbar neuritis, herpes zoster, migraine, and leaking aneurysm can all occur in the absence of physical findings in the early stages.

Increasing clinical maturity will allow the recognition of "top dressing" anxiety or "hysterical" reactions of patients suffering severe symptomatology.

Primary psychogenic symptomatology may be an easy diagnosis as, for example, the chronic aching of both eyes in the elderly patient admitting depression and isolation. Likewise, it may be easy to miss an organic presentation in a previously "hysteroid"frequent attender. Careful history taking, eliminating the chaff from the wheat, and pertinent examination remain as important in ophthalmology as in general medicine.

Interestingly, "morbid periodicity" certainly occurs where the symptom appears on the anniversary of an emotionally traumatic event. ${ }^{4}$ Mimicry of symptoms of the patient's nearest and dearest such as headache from a feared cerebral tumour, also occurs as does the engendering of symptoms from simple fatigue, mental or physical; worry about a life situation; or the state of eye health. Such phenomena clearly occur across the entire spectrum of medicine but are possibly less appreciated in the "high tech" area of ophthalmology. ${ }^{5-7}$ It is, however, an area of medicine that does not change much with time!

\section{Dysthyroid disease}

Ophthalmic involvement may be the presenting feature. Periorbital subcutaneous swelling of the upper and lower lids and/or upper lid retraction are the commonest changes.

Sclera showing above the cornea in the primary position is often bilateral and symmetrical, indicating upper lid retraction. It may be
1 Hansson L, Zanchetti A, Carruthers SG, et al. Effects of intensive blood pressure lowering and low dose aspirin in patients with hypertension. Principal results of the hypertension optimal treatment randomised trial. HOT Study tension optimal treatment randornis. Lancet 1998;351:1755-62.

2 Broup. Lancet 1998;351:1755-62.

3 Backhouse O, Parapia L, Mahomed I, et al. Familial thrombophilia and retinal vein occlusion. Eye 2000;14:13. 
4 Inman WS. Clinical observations on morbid periodicity. $\mathrm{Br}$ f Med Psychol 1948;XXI:254-62.

5 Karseras AG. Psychosomatic ophthalmology. In: Howells $\mathrm{JG}$, ed. Modern perspectives in the psychiatric aspects of surgery. New York: Brunner/Mazel, 1976: 206-24.
6 Crisp AH, Karseras AG. Symptoms without signs in outpatients attending ophthalmology clinics. Br $\mathscr{f}$ Ophthalmol tients attending

7 Karseras AG, Sullivan S. Ophthalmic psychoneurosis: natural history. Br F Ophthalmol 1981;67:554-6.

\section{IMAGES IN MEDICINE}

\section{Multifocal breast cancer}

Computed tomography is not a usual primary imaging modality for examining breast abnormalities. Mammography and ultrasonography are the standard techniques with magnetic resonance imaging reserved for equivocal cases such as possible post-treatment recurrence. ${ }^{1}$ This patient underwent thoracic computed tomography (see fig 1) and was found to have multiple dense lesions in the right breast. Subsequent histological examinations of specimens from core biopsy and mastectomy confirmed multifocal lobular carcinoma.

K S HINDLE Luton and Dunstable Hospital

A HUANG

Chelsea and Westminster Hospital Correspondence to: $M r$ A Huang, 40 York Terrace East, Regent's Park, London NW1 4PT, UK email:andyhuang@talk21.com

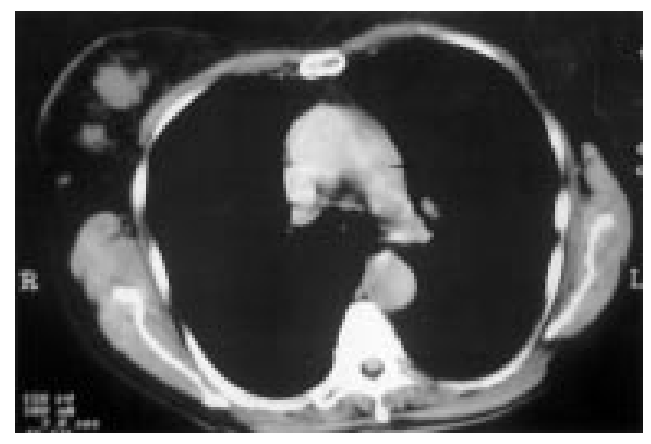

Figure 1 Computed tomogram of patient.

1 Mumtaz H, Davidson T, Hall-Craggs MA, et al. Comparison of magnetic resonance imaging and conventional triple
assessment in locally recurrent breast cancer. $\mathrm{Br} f$ Surg 1997;84:1147-51. 